\title{
Halving dynamical systems
}

\author{
Shaun Stevens, Tom Ward, and Stefanie Zegowitz
}

\begin{abstract}
We discuss the possible orbit-growth behaviour in pairs of topological dynamical systems one of which is obtained from the other by forming the quotient under the action of an involution. Examples illustrate the extent to which their orbit behaviour can differ.
\end{abstract}

\section{Introduction.}

Our purpose here is to discuss doubling and halving in the context of topological dynamical systems, which for convenience we take to mean pairs $(X, T)$ where $X$ is a compact metric space and $T: X \rightarrow X$ is a homeomorphism with a fixed point and with finitely many points of period $n$ for each $n \geqslant 1$. Halving (and doubling) may be thought of as a relationship between pairs $(X, T)$ and $(\bar{X}, \bar{T})$ of the following form. Suppose there is a continuous involution $\imath: X \rightarrow X$ that commutes with $T$, and use this to define an equivalence relation on $X$ by saying that $x \sim y$ if and only if $x=\imath(y)$. Writing $[x]_{\sim}$ for the equivalence class $\{x, \imath(x)\}$ of $x$ we define $\bar{X}$ to be the quotient space $X / \sim$ and $\bar{T}$ to be the map defined by $\bar{T}\left([x]_{\sim}\right)=[T(x)]_{\sim}$. The process of passing from $(X, T)$ to $(\bar{X}, \bar{T})$ may be thought of as halving, and passing from $(\bar{X}, \bar{T})$ to $(X, T)$ as doubling.

The question we address is to ask which structures are preserved and which are not by doubling and halving in this sense. The most important quantity associated to a topological dynamical system is the topological entropy, which will not be of interest here as it is preserved by halving (see [3, Ch. 5] for the definition and details). We are particularly interested in the relationship between closed orbits in the two systems. It turns out that there are some constraints on the relationship between the numbers of closed orbits for $T$ and for $\bar{T}$ but that, within these constraints, essentially everything is possible. The constraints will be described in Lemma 5, and the freedom within those constraints in Corollary 10.

\section{Closed orbits}

We begin with some notational conventions for a map $T: X \rightarrow X$. For $n$ a natural number, we write $\mathscr{F}_{T}(n)=\left\{x \in X \mid T^{n}(x)=x\right\}$ for the set of points of period $n$ under iteration of $T$, and $\mathrm{F}_{T}(n)=\left|\mathscr{F}_{T}(n)\right|$ for the number of points of period $n$. Similarly, write $\mathscr{O}_{T}(n)$ for the set of closed orbits of length $n$ under

2000 Mathematics Subject Classification. Primary 37P35, 54H20. 
iteration of $T$, and $O_{T}(n)=\left|\mathscr{O}_{T}(n)\right|$ for the number of closed orbits of length $n$. The set of points of period $n$ comprises exactly the disjoint union of those points on a closed orbit of length $d$ for each $d$ dividing $n$, and each such orbit consists of $d$ distinct points. Thus

$$
\mathrm{F}_{T}(n)=\sum_{d \mid n} d \mathrm{O}_{T}(d)
$$

and hence, by Möbius inversion,

$$
\mathrm{O}_{T}(n)=\frac{1}{n} \sum_{d \mid n} \mu\left(\frac{n}{d}\right) \mathrm{F}_{T}(d)
$$

for any $n \geqslant 1$.

A convenient generating function for the periodic point data is the dynamical zeta function

$$
\zeta_{T}(z)=\exp \left(\sum_{n \geqslant 1} \mathrm{~F}_{T}(n) \frac{z^{n}}{n}\right)=\prod_{n \geqslant 1}\left(1-z^{n}\right)^{-\mathrm{O}_{T}(n)},
$$

(the second equality is equivalent to the identity (1)), which defines a function under the assumption that $\mathrm{F}_{T}(n)<\infty$ for all $n \geqslant 1$, in which case it has radius of convergence given by

$$
\left(\limsup _{n \rightarrow \infty}\left(\mathrm{F}_{T}(n)\right)^{1 / n}\right)^{-1}
$$

\section{Closed orbits and topological factors}

If $(X, T)$ and $(Y, S)$ are topological dynamical systems with a continuous surjective map $\pi: X \rightarrow Y$ satisfying $\pi \circ T=S \circ \pi$, then $(Y, S)$ is called a topological factor of $(X, T)$, and halving is a special case of this. In general, closed orbits can behave very badly under a topological factor map, as the following examples illustrate.

Example 1. Closed orbits in $(X, T)$ may be glued together in a topological factor. An extreme instance of this is to take $Y=\{y\}$ to be a singleton, set $S(y)=y$, and define the factor map $\pi$ by $\pi(x)=y$ for all $x \in X$. Then, whatever the sequence $\left(\mathrm{O}_{T}(n)\right)$ of numbers of closed orbits under $T$, the factor system has $\mathrm{O}_{S}(1)=1$ and $\mathrm{O}_{S}(n)=0$ for all $n \geqslant 2$.

EXAmple 2. Finite pieces of orbits in $(X, T)$ that are not closed may close up under the factor map, producing closed orbits in $(Y, S)$. For example, take any topological dynamical system $(Y, S)$ and form the product system $X=Y \times \mathbb{T}$, where $\mathbb{T}=\mathbb{R} / \mathbb{Z}$ is the additive circle, with map $T(y, t)=(S(y), t+\alpha(\bmod 1))$ for some fixed $\alpha \notin \mathbb{Q}$. Then $(Y, S)$ is a topological factor of $(X, T)$ via the projection onto the first coordinate. Clearly whatever the sequence $\left(\mathrm{O}_{S}(n)\right)$ of numbers of closed orbits under $S$, the map $T$ has no orbits of finite length.

For quotienting by an action of $C_{2}$ (that is, halving), the situation is more restricted.

Example 3 . Let $X=\mathbb{T}=\mathbb{R} / \mathbb{Z}$ be the additive circle, and define the map $T$ on $X$ by $T(x)=2 x(\bmod 1)$. This is not a homeomorphism, but is a convenient familiar map to use as an initial example. The involution $\imath(x)=1-x$ commutes 
with $T$, and so defines a halved system $(\bar{X}, \bar{T})$. A convenient way to visualize this system is to imagine looking sideways at the unit circle so that points identified by the map $\imath$ are seen as a single point, as illustrated in Figure 1. It is straightforward

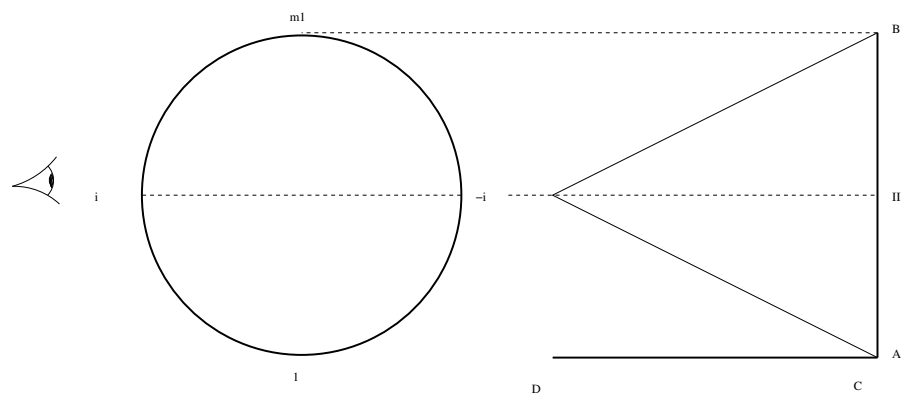

Figure 1. Halving the circle doubling map gives a tent map.

to check that the quotient system $(\bar{X}, \bar{T})$ is the tent map, with $\bar{X}=\left[0, \frac{1}{2}\right]$ and

$$
\bar{T}(x)= \begin{cases}2 x & 0 \leqslant x \leqslant \frac{1}{4}, \\ 1-2 x & \frac{1}{4} \leqslant x \leqslant \frac{1}{2} .\end{cases}
$$

For these two systems it is easy to calculate that

$$
\zeta_{T}(z)=\frac{1-z}{1-2 z}
$$

and

$$
\zeta_{\bar{T}}(z)=\frac{1}{1-2 z}
$$

In this example, a system with approximately $2^{n}$ points of period $n$ and a rational zeta function is halved to a system with the same properties.

The following example gives a natural way in which one could double a topological dynamical system, simply by putting together two copies of the system.

ExAmple 4. Given any dynamical system $(Y, S)$ on a metric space $(Y, d)$ we may form the doubled space $X=Y \times\{0,1\}$ and define a map on the doubled space by

$$
\begin{aligned}
T: X & \longrightarrow X \\
(y, e) & \longmapsto(S(y), e+1 \quad(\bmod 2)) .
\end{aligned}
$$

The involution $\imath:(y, e) \longmapsto(y, e+1(\bmod 2))$ commutes with $T$, giving a halved system $(\bar{X}, \bar{T})$ which can be identified with the original system $(Y, S)$. Clearly

$$
\mathrm{F}_{T}(n)= \begin{cases}0 & \text { if } n \text { is odd } \\ 2 \mathrm{~F}_{S}(n) & \text { if } n \text { is even }\end{cases}
$$

so that

$$
\limsup _{n \rightarrow \infty} \frac{1}{n} \log \mathrm{F}_{T}(n)=\limsup _{n \rightarrow \infty} \frac{1}{n} \log \mathrm{F}_{S}(n),
$$

meaning that the zeta functions $\zeta_{T}$ and $\zeta_{\bar{T}}=\zeta_{S}$ have the same radius of convergence. Moreover, the relation (2) may be written as

$$
\zeta_{T}(s)=\zeta_{S}(s) \zeta_{S}(-s)
$$


showing that if $\zeta_{S}$ is rational then $\zeta_{T}$ is also rational, though we will see later that the converse is not true (see Example 11).

Our purpose here is to show how extremely unrepresentative Examples 3 and 4 really are. In general, both the growth rate in the number of periodic points and the arithmetic nature of the zeta function do not survive under doubling or halving. However, in contrast to Examples 1 and 2 we will show that the change of the growth rate in the number of closed orbits is restricted.

\section{Shortening, Surviving, and Gluing Orbits}

We put ourselves back in the halving situation of the introduction. Thus $(X, T)$ is a topological dynamical system, which we recall means a pair with $X$ a compact metric space and $T: X \rightarrow X$ a homeomorphism with a fixed point and with finitely many points of period $n$ for each $n \geqslant 1$, and $\imath$ is a continuous involution on $X$ which commutes with $T$. Then $\bar{X}$ is the quotient of $X$ under the equivalence relation induced by $\imath$, with quotient map $\pi: X \mapsto \bar{X}$ given by $\pi(x)=\{x, \imath(x)\}$. Note that $\bar{X}$ is a metric space: if $d_{X}$ is the metric on $X$, then the metric $d_{\bar{X}}$ on $\bar{X}$ is given by

$$
d_{\bar{X}}(\bar{x}, \bar{y})=\min \left\{d_{X}(x, y) \mid x \in \pi^{-1}(\bar{x}), y \in \pi^{-1}(\bar{y})\right\} .
$$

The map $\bar{T}: \bar{X} \rightarrow \bar{X}$ is (well-)defined by the relation $\pi \circ T=\bar{T} \circ \pi$, and is a homeomorphism.

The factor map $\pi$ maps any closed orbit under $T$ to a closed orbit under $\bar{T}$; conversely, since the fibres of $\pi$ are finite, the inverse image under $\pi$ of a closed orbit under $\bar{T}$ is a finite set closed under $T$ so is a finite union of closed orbits under $T$. In particular, $\pi$ induces a surjective map

$$
\bigsqcup_{n=1}^{\infty} \mathscr{O}_{T}(n) \rightarrow \bigsqcup_{n=1}^{\infty} \mathscr{O}_{\bar{T}}(n)
$$

In order to analyze this more closely, let $\tau=\left\{x, T(x), T^{2}(x), \ldots, T^{n}(x)=x\right\}$ be a closed orbit in $(X, T)$ of length $n$. Then $\imath$ and $\tau$ can interact in just three ways.

(1) It could fix the orbit $\tau$ pointwise, (we say that $\tau$ 'survives'), that is $x=\imath(x)$ for all $x \in \tau$; we write $\mathscr{O}_{T}^{S}(n)$ for the set of closed orbits of length $n$ under $T$ that are fixed pointwise by $\imath$. Then the factor map $\pi$ induces an injective $\operatorname{map} \mathscr{O}_{T}^{s}(n) \rightarrow \mathscr{O}_{\bar{T}}(n)$.

(2) It could map $\tau$ to another closed orbit $\tau^{\prime}$ of the same length (we say that $\tau$ is 'glued' to $\tau^{\prime}$ ), that is $\imath(x) \in \tau^{\prime}$ for all $x \in \tau$; we write $\mathscr{O}_{T}^{g}(n)$ for the set of closed orbits of length $n$ under $T$ that are glued together in pairs. The factor map $\pi$ induces a 2-to-1 map $\mathscr{O}_{T}^{g}(n) \rightarrow \mathscr{O}_{\bar{T}}(n)$.

(3) It could preserve the orbit $\tau$ but not fix it pointwise; in that case, we must have $n=2 k$ even and $\imath(x)=T^{k}(x)$, for all $x \in \tau$, so that the orbit $\pi(\tau)$ of $\bar{T}$ has length $k$ (we say that $\tau$ is 'halved'). we write $\mathscr{O}_{T}^{h}(n)$ for the set of closed orbits of length $n$ under $T$ that are halved in length, and $\pi$ induces an injective $\operatorname{map} \mathscr{O}_{T}^{h}(2 k) \rightarrow \mathscr{O}_{\bar{T}}(k)$.

Clearly

$$
\mathscr{O}_{T}(n)=\mathscr{O}_{T}^{h}(n) \sqcup \mathscr{O}_{T}^{g}(n) \sqcup \mathscr{O}_{T}^{s}(n)
$$


is a disjoint union, and so

$$
\mathrm{O}_{T}(n)=\mathrm{O}_{T}^{h}(n)+\mathrm{O}_{T}^{g}(n)+\mathrm{O}_{T}^{s}(n),
$$

for any $n \geqslant 1$. Similarly, from the surjective map (4) and the three possible behaviours, we get

$$
\mathscr{O}_{\bar{T}}(n)=\pi\left(\mathscr{O}_{T}^{h}(2 n)\right) \sqcup \pi\left(\mathscr{O}_{T}^{g}(n)\right) \sqcup \pi\left(\mathscr{O}_{T}^{s}(n)\right),
$$

and it follows that

$$
\mathrm{O}_{\bar{T}}(n)=\mathrm{O}_{T}^{h}(2 n)+\frac{1}{2} \mathrm{O}_{T}^{g}(n)+\mathrm{O}_{T}^{s}(n),
$$

for any $n \geqslant 1$. Since these numbers are finite and

$$
\mathrm{O}_{\bar{T}}(1) \geqslant \mathrm{O}_{T}(1)-\frac{1}{2} \mathrm{O}_{T}^{g}(1) \geqslant \frac{1}{2} \mathrm{O}_{T}(1)>0,
$$

we see that $(\bar{X}, \bar{T})$ is also a topological dynamical system.

The way in which the set of orbits of length $n$ under $T$ decomposes into those that halve in length, those that glue together, and those that survive, is not arbitrary. Whatever constraints on $\imath$ arise from having to be a continuous involution on $X$ that commutes with $T$, there are some purely combinatorial constraints as follows:

$$
\begin{gathered}
\mathrm{O}_{T}^{h}(n)=0 \text { if } n \text { is odd; } \\
\mathrm{O}_{T}^{g}(n) \text { is even for all } n \geqslant 1 .
\end{gathered}
$$

These observations already constrain the effect that halving can have on the growth rate of closed orbits.

Lemma 5. Suppose $(\bar{X}, \bar{T})$ is obtained from $(X, T)$ by halving. Then, for any $n \geqslant 1$,

(a) $\frac{1}{2} \mathrm{~F}_{T}(n) \leqslant \mathrm{F}_{\bar{T}}(n) \leqslant \frac{1}{2}\left(\mathrm{~F}_{T}(n)+\mathrm{F}_{T}(2 n)\right)$;

(b) $\mathrm{O}_{\bar{T}}(n) \leqslant \mathrm{O}_{T}(n)+\mathrm{O}_{T}(2 n)$, and if $n$ is odd then $\mathrm{O}_{\bar{T}}(n) \geqslant \frac{1}{2} \mathrm{O}_{T}(n)$.

Proof. (a) The lower bound for $\mathrm{F}_{\bar{T}}(n)$ comes from the fact that the fibres of the factor map $\pi$ have cardinality at most 2 ; since $\pi$ maps $\mathscr{F}_{T}(n)$ to $\mathscr{F}_{\bar{T}}(n)$, we deduce that $\mathrm{F}_{\bar{T}}(n) \geqslant \frac{1}{2} \mathrm{~F}_{T}(n)$. The bound is achieved if all orbits of length dividing $n$ glue together in pairs. The upper bound comes from the containment $\pi^{-1}\left(\mathscr{F}_{T}(n)\right) \subseteq \mathscr{F}_{T}(2 n)$. If $x \in \mathscr{F}_{T}(2 n) \backslash \mathscr{F}_{T}(n)$, then $\pi(x) \in \mathscr{F}_{T}(n)$ if and only if $x$ lies in an orbit which halves in length, in which case $x$ lies in a fibre of cardinality 2. Thus

$$
\begin{aligned}
\mathrm{F}_{\bar{T}}(n) & =\left|\mathscr{F}_{\bar{T}}(n) \cap \pi\left(\mathscr{F}_{T}(2 n) \backslash \mathscr{F}_{T}(n)\right)\right|+\left|\mathscr{F}_{\bar{T}}(n) \cap \pi\left(\mathscr{F}_{T}(n)\right)\right| \\
& \leqslant \frac{1}{2}\left(\mathrm{~F}_{T}(2 n)-\mathrm{F}_{T}(n)\right)+\mathrm{F}_{T}(n) \\
& =\frac{1}{2}\left(\mathrm{~F}_{T}(2 n)+\mathrm{F}_{T}(n)\right) .
\end{aligned}
$$

The upper bound is achieved if all orbits of order dividing $n$ survive, while all other orbits of order dividing $2 n$ halve.

(b) For the upper bound for $\mathrm{O}_{\bar{T}}(n)$, we have

$$
\mathrm{O}_{\bar{T}}(n)=\mathrm{O}_{T}^{h}(2 n)+\frac{1}{2} \mathrm{O}_{T}^{g}(n)+\mathrm{O}_{T}^{s}(n) \leqslant \mathrm{O}_{T}(2 n)+\mathrm{O}_{T}(n),
$$

by (5) and (6). Again this is achieved when all orbits of order $n$ survive, while all orbits of order $2 n$ halve. 
For the lower bound, when $n$ is odd we have $\mathrm{O}_{T}^{h}(n)=0$ so that

$$
\mathrm{O}_{T}(n)=\mathrm{O}_{T}^{s}(n)+\mathrm{O}_{T}^{g}(n)
$$

and, from (6),

$$
\mathrm{O}_{\bar{T}}(n) \geqslant \frac{1}{2} \mathrm{O}_{T}^{g}(n)+\mathrm{O}_{T}^{s}(n) \geqslant \frac{1}{2} \mathrm{O}_{T}(n) .
$$

This bound is achieved if all orbits of length $n$ are glued in pairs.

Remark 6. Note that Lemma 5 does not give a lower bound for $\mathrm{O}_{\bar{T}}(n)$ when $n$ is even. Specifically, when $n$ is even, all orbits of length $n$ might halve in length while orbits of length $2 n$ retain their length. Thus the only possible lower bound is the trivial one $\mathrm{O}_{\bar{T}}(n) \geqslant 0$.

In the case that $\mathrm{O}_{T}(n)$ grow exponentially, Lemma 5 immediately gives bounds on the logarithmic growth rate of $\mathrm{O}_{\bar{T}}(n)$.

Corollary 7. Let $(X, T)$ be a topological dynamical system, let $\imath$ be a continuous involution on $X$ commuting with $T$, and let $(\bar{X}, \bar{T})$ be the halved system. Suppose there is a real number $\lambda>0$ such that $\limsup _{n \rightarrow \infty} \frac{1}{n} \log \mathrm{F}_{T}(n)=\lambda$. Then

$$
\lambda \leqslant \limsup _{n \rightarrow \infty} \frac{1}{n} \log \mathrm{F}_{\bar{T}}(n) \leqslant 2 \lambda .
$$

We will see later (see Corollary 10) that every growth rate in the closed interval $[\lambda, 2 \lambda]$ is obtainable.

\section{The basic lemma}

Our main observation is that, if we are free to choose the topological dynamical systems, then (7) and (8) are the only constraints on the behaviour of closed orbits under halving. This is a simple extension of an elementary remark in [7]: for any sequence $\left(a_{n}\right)$ of non-negative integers, there is a topological dynamical system $(X, T)$ with $\mathrm{O}_{T}(n)=a_{n}$ for all $n \geqslant 1$.

LEMma 8. Let $\left(b_{n}^{s}\right),\left(b_{n}^{g}\right)$, and $\left(b_{n}^{h}\right)$ be three sequences of non-negative integers, with $b_{1}^{s}>0$. Define sequences $\left(a_{n}^{h}\right),\left(a_{n}^{g}\right)$, and $\left(a_{n}^{s}\right)$ by

$$
a_{n}^{s}=b_{n}^{s}, \quad a_{n}^{g}=2 b_{n}^{g}, \quad \text { and } \quad a_{n}^{h}= \begin{cases}b_{n / 2}^{h} & \text { if } n \text { is even } \\ 0 & \text { otherwise. }\end{cases}
$$

Define $a_{n}=a_{n}^{s}+a_{n}^{g}+a_{n}^{h}$ and $b_{n}=b_{n}^{s}+b_{n}^{g}+b_{n}^{h}$ for all $n \geqslant 1$. Then there are $a$ topological dynamical system $(X, T)$ and a continuous involution $\imath: X \rightarrow X$ that commutes with $T$, such that $\mathrm{O}_{T}(n)=a_{n}$ and $\mathrm{O}_{\bar{T}}(n)=b_{n}$, for all $n \geqslant 1$.

Notice in particular that taking $b_{n}^{s}=a_{n}$ and $b_{n}^{g}=b_{n}^{h}=0$, for all $n \geqslant 1$ recovers the basic lemma of [7].

Before giving an algebraic proof, we give a more geometric sketch to give an idea of what is happening. We can construct $X$ as a closed (and hence compact) subset of the triangle $\{(x, y) \mid 0 \leqslant x \leqslant 1,0 \leqslant y \leqslant x\} \subset \mathbb{R}^{2}$, with the metric inherited from $\mathbb{R}^{2}$. Above each point $\left(\frac{1}{n}, 0\right)$ for $n \geqslant 2$ draw $a_{n}$ disjoint regular $n$-gons in such a way that all of them are disjoint as subsets of the plane. By hypothesis $a_{1} \geqslant 1$, and we draw $a_{1}-1$ points (1-gons) above $(1,0)$. Finally locate a single 1 -gon at $(0,0)$ (which may be thought of as a point 'at infinity'). The space $X$ is now defined to be the union of all the vertices of the polygons. (See Figure 2.) It is closed because all but one point is isolated, and the accumulation point $(0,0)$ is, by construction, 
a member of $X$. Number the vertices of each $n$-gon with the numbers 1 to $n$ consecutively clockwise, so that we may speak of the 'same' point on two disjoint $n$ gons as being the point with the same symbol. The homeomorphism $T$ is defined to be the map that takes each point on any $n$-gon to the next point in a clockwise orientation around the same $n$-gon (equivalently, adding one using the labels; the action of this map is illustrated by the lines joining vertices of the polygons in Figure 2). This defines a homeomorphism since all but one point is isolated in $X$, and all the points close to the fixed point $(0,0)$ are moved by a very small distance. Then $(X, T)$ is a topological dynamical system, and by construction $\mathrm{O}_{T}(n)=a_{n}$ for all $n \geqslant 1$.

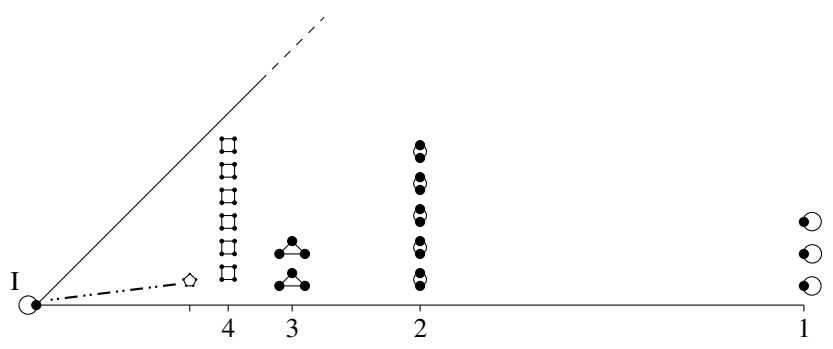

Figure 2. Building the system $(X, T)$.

Now we define an action of $C_{2}$ on $X$ using the numbers $a_{n}^{s}, a_{n}^{g}$, and $a_{n}^{h}$ as follows.

- For each $n \geqslant 1$ pick $\frac{1}{2} a_{n}^{g}$ pairs of $n$-gons above $\left(\frac{1}{n}, 0\right)$ and define the action of $\imath$ to send a point on any one of them to the same point on the paired $n$-gon (these are the glued orbits).

- For each $n \geqslant 1$ pick $a_{n}^{h}$ of the $n$-gons above $\left(\frac{1}{n}, 0\right)$, chosen from those that have not been chosen already for gluing, and on each polygon (which will by hypothesis have even length) define the action of $\imath$ to be rotation by $\pi$ (these are the halved orbits).

- On all the remaining points that are vertices of polygons that are neither glued nor halved, define $\imath$ to be the identity map (these are the surviving orbits).

It is clear that $\imath$ is continuous (since all points close to the fixed point are moved by a very small distance) and commutes with $T$ and so defines a halved system $(\bar{X}, \bar{T})$ which, by construction, has the required numbers of orbits.

Note that, in the proof below, we do not refer to the triangle in the plane and the metric on $X$ we give is not the same one as in this sketch, but it does give the same topology.

Proof of Lemma 8. We write $C_{2}=\{e, \imath\}$, where $e$ is the identity element, for the cyclic group of order 2 . We begin by describing $X$ as a set, before compactifying. It will take the form

$$
X=\bigsqcup_{n \geqslant 1} X_{n},
$$


where each $X_{n}$ will be the union of closed orbits of length $n$. We set

$$
X_{n}=X_{n}^{s} \sqcup X_{n}^{g} \sqcup X_{n}^{h}, \quad \text { where }\left\{\begin{array}{l}
X_{n}^{s}=\left\{1,2, \ldots, a_{n}^{s}\right\} \times \mathbb{Z} / n \mathbb{Z}, \\
X_{n}^{g}=\left\{1,2, \ldots, b_{n}^{g}\right\} \times C_{2} \times \mathbb{Z} / n \mathbb{Z}, \\
X_{n}^{h}=\left\{1,2, \ldots, a_{n}^{h}\right\} \times \mathbb{Z} / n \mathbb{Z} .
\end{array}\right.
$$

We define $T: X \rightarrow X$ and $\imath: X \rightarrow X$ by describing their restrictions to each of the sets $X_{n}^{s}, X_{n}^{g}, X_{n}^{h}$, which will be preserved:

- for $x=(i, k) \in X_{n}^{s}$, put $T(x)=(i, k+1(\bmod n))$ and $\imath(x)=x$;

- for $x=(i, \gamma, k) \in X_{n}^{g}$, put $T(x)=(i, \gamma, k+1(\bmod n))$ and $\imath(x)=$ $(i, \imath \gamma, k)$

- for $x=(i, k) \in X_{n}^{h}$, so that $n$ is even, we put $T(x)=(i, k+1(\bmod n))$ and $\imath(x)=\left(i, k+\frac{n}{2}(\bmod n)\right)$.

Then $\imath$ commutes with $T$ and, by construction, the map $T$ and the induced map $\bar{T}$ on the quotient set $\bar{X}$ have the required numbers of orbits.

It remains to show that $X$ can be given the structure of a compact metric space, with respect to which $T$ and $\imath$ are homeomorphisms. To do this, we pick a point in $X_{1}^{s}$ (which is non-empty by hypothesis) and call it $\infty$ and define a metric as follows: if $x \in X_{m}$ and $y \in X_{n}$, with $x \notin y, \infty$, then

$$
d(x, y)=d(y, x)= \begin{cases}\frac{1}{m} & \text { if } y=\infty \\ \frac{1}{\min \{m, n\}} & \text { otherwise }\end{cases}
$$

and $d(x, x)=0$. It is straightforward to check that this does indeed define a metric and, given any open set $U$ containing $\infty$, there exists $N \geqslant 1$ such that $U$ contains $\bigsqcup_{n \geqslant N} X_{n}$ so that $X \backslash U$ is finite; hence $X$ is compact. Moreover, since $T$ and $\imath$ preserve the sets $X_{n}$ and the point $\infty$, they are isometries, so homeomorphisms, and we are done.

\section{Growth in closed orbits}

Lemma 8 shows that any pair of sequence $\left(a_{n}\right),\left(b_{n}\right)$ for which the combinatorial constraints (5)-(8) are satisfied does in fact arise as the orbit count of a pair of systems related by halving. However, it is not so easy to give conditions directly on the sequences $\left(a_{n}\right),\left(b_{n}\right)$ which guarantee that the combinatorial constraints are indeed satisfied. The following result gives some sufficient conditions.

Proposition 9. Let $\left(a_{n}\right)$ be a sequence of non-negative integers with $a_{1} \geqslant 1$ such that there is an integer $N \geqslant 1$ with $a_{2 n} \geqslant \frac{1}{2} a_{n}$, for all $n \geqslant N$. Let $\left(b_{n}\right)$ be any sequence of integers such that $b_{1}>\frac{1}{2} a_{1}$ and

$$
\begin{cases}\frac{1}{2} a_{n} \leqslant b_{n} \leqslant a_{n} & \text { for } n<N \\ \frac{1}{2} a_{n} \leqslant b_{n} \leqslant a_{2 n} & \text { for } n \geqslant N .\end{cases}
$$

Then there is a topological dynamical system $(X, T)$ with a continuous involution $\imath$ of $X$ commuting with $T$, such that $\mathrm{O}_{T}(n)=a_{n}$ and $\mathrm{O}_{\bar{T}}(n)=b_{n}$, for all $n \geqslant 1$.

The conditions on the pair of sequences $\left(a_{n}\right),\left(b_{n}\right)$ here are not necessary for the existence of a suitable halving system, but they are sufficient for our interests and are not so far from being necessary: for $n>N$ odd, the condition

$$
\frac{1}{2} a_{n} \leqslant b_{n} \leqslant a_{2 n}
$$

is necessary by Lemma 5 . 
Proof. In order to use Lemma 8 , we recursively define non-negative integers $b_{n}^{s}, b_{n}^{g}$ and $b_{n}^{h}$ such that

$$
b_{n}=b_{n}^{s}+b_{n}^{g}+b_{n}^{h}, \quad a_{n}=b_{n}^{s}+2 b_{n}^{g}+b_{n / 2}^{h}, \quad \text { and } \quad b_{n}^{h} \leqslant a_{2 n},
$$

where we understand $b_{n / 2}^{h}=0$ when $n / 2 \notin \mathbb{Z}$. So suppose $k \geqslant 1$ and we have defined these for $n<k$. Then there are two cases.

If $b_{k} \leqslant a_{k}-b_{k / 2}^{h}$, which we note is always the case for $k<N$, then we put

$$
b_{k}^{g}=a_{k}-b_{k}-b_{k / 2}^{h}, \quad b_{k}^{s}=b_{k}-b_{k}^{g}, \quad b_{k}^{h}=0 .
$$

On the other hand, if $b_{k}>a_{k}-b_{k / 2}^{h}$, then we put

$$
b_{k}^{g}=0, \quad b_{k}^{s}=a_{k}-b_{k / 2}^{h}, \quad b_{k}^{h}=b_{k}-b_{k}^{s} .
$$

These are non-negative and, in the latter case, we have $b_{k}^{h} \leqslant b_{k} \leqslant a_{2 k}$, since $k \geqslant N$. Note also that, in either case, $b_{1}^{s}>0$. Now Lemma 8 implies the result.

As a consequence, we get the following result when we consider exponential orbit growth rates.

COROLlary 10. Let $\lambda, \eta, c$ be positive real numbers with $\lambda>1$ and

$$
\left\{\begin{array}{l}
\eta=\lambda \text { and } c \geqslant \frac{1}{2}, \text { or } \\
\eta \in\left(\lambda, \lambda^{2}\right), \text { or } \\
\eta=\lambda^{2} \text { and } 0<c \leqslant 1
\end{array}\right.
$$

Then there exist a topological dynamical system $(X, T)$ and an involution $\imath$ on $X$ commuting with $T$, such that

$$
\mathrm{O}_{T}(n) \sim \lambda^{n} \quad \text { and } \quad \mathrm{O}_{\bar{T}}(n) \sim c \eta^{n} \quad \text { as } n \rightarrow \infty .
$$

Proof. Let $N>1$ be any integer such that $c \eta^{N}<\lambda^{2 N}$ and define sequences by

$$
a_{n}=\left\lceil\lambda^{n}\right\rceil, \quad b_{n}= \begin{cases}a_{n} & \text { if } n<N, \\ \left\lceil c \eta^{n}\right\rceil & \text { if } n \geqslant N,\end{cases}
$$

for $n \geqslant 1$. This gives a pair of sequences satisfying the hypotheses of Proposition 9, from which the result follows.

\section{Dynamical zeta functions}

Bowen and Lanford [1, Th. 2] showed that there are only countably many rational dynamical zeta functions, so Corollary 10 shows in particular that halving and doubling cannot preserve the property of having a rational zeta function. We now discuss some examples that give concrete instances of this phenomenon. The arguments all rely on the following facts: a power series with positive radius of convergence represents a rational function if and only if the coefficients satisfy a linear recurrence (see [4, Sec.1.1]). Moreover, the Skolem-Mahler-Lech Theorem says that, in any linear recurrence sequence $\left(a_{n}\right)$, the set of zeros, comprising those values of $n \in \mathbb{N}$ for which $a_{n}=0$, is the union of a finite set of arithmetic progressions and a finite set (see [4, Ch. 1] for further details and a proof). 
ExAmple 11. We revisit Example 4, so that $(Y, S)$ is a topological dynamical system and $X=Y \times\{0,1\}$ with the map $T(y, e)=(S(y), e+1(\bmod 2))$. The involution $\imath:(y, e)=(y, e+1(\bmod 2))$ commutes with $T$, giving the halved system $(\bar{X}, \bar{T})=(Y, S)$. There is sufficient freedom in the choice of orbits of odd length under $S$ to allow us to find examples with $\zeta_{\bar{T}}=\zeta_{S}$ irrational but $\zeta_{T}$ rational. In particular, we may take

$$
\mathrm{F}_{S}(n)= \begin{cases}2^{n}+1 & \text { if } n \text { is even } \\ \sum_{d \mid n} d 2^{(d-1) / 2} & \text { if } n \text { is odd. }\end{cases}
$$

It is a pleasant exercise to verify that these really do arise from a topological dynamical system (that is, $\frac{1}{n} \mathrm{O}_{S}(n)$ is a non-negative integer for each $n$ ). Then by $(2)$

$$
\zeta_{T}(z)=\frac{1}{\left(1-z^{2}\right)\left(1-4 z^{2}\right)}
$$

is rational. On the other hand

$$
z \frac{\zeta_{S}^{\prime}(z)}{\zeta_{S}(z)}=\sum_{n=1}^{\infty} \mathrm{F}_{S}(n) z^{n}=\frac{z}{1-z}+\frac{4 z^{2}}{1-4 z^{2}}+\frac{6 z^{3}-4 z^{5}}{\left(1-2 z^{2}\right)^{2}}+\varphi(z)
$$

where

$$
\varphi(z)=\sum_{n=1}^{\infty} z^{2 n+1} \sum_{\begin{array}{c}
d \mid 2 n+1 \\
d \neq 1,2 n+1
\end{array}} d 2^{(d-1) / 2} .
$$

We claim that $\varphi$ is irrational, so that $\zeta_{S}$ is irrational. To see this, note that the coefficient of $z^{n}$ in $\varphi(z)$ vanishes precisely when $n$ is even or $n$ is an odd prime; since the set of primes is infinite, while any arithmetic progression contains composites, the Skolem-Mahler-Lech Theorem implies the sequence of coefficients cannot be a linear recurrence sequence and hence $\varphi$ cannot be a rational function.

Our next examples use the sum of divisors function $\sigma(n)=\sum_{d \mid n} d$. There are sophisticated bounds for size of $\sigma(n)$, but for our purposes it is sufficient to note the trivial bounds

$$
n \leqslant \sigma(n) \leqslant n^{2} .
$$

It follows that the complex power series

$$
\theta(z)=\exp \sum_{n \geqslant 1} \sigma(n) \frac{z^{n}}{n}
$$

has radius of convergence 1 . It is known that

$$
\frac{1}{\theta(z)}=1-z-z^{2}+z^{5}+z^{7}-\cdots,
$$

where the powers of $z$ are those of the form $\left(3 k^{2} \pm k\right) / 2$ (see, for example, Pólya and Szegö [6, Sec. VIII, Ex. 75]). This means that $\frac{1}{\theta(z)}$ is a power series with arbitrarily long consecutive sequences of zero coefficients. Thus, by the Skolem-Mahler-Lech Theorem, the coefficients of $\frac{1}{\theta(z)}$ are not a linear recurrence sequence and we deduce that $\theta(z)$ is not a rational function of $z$. 
EXAMPLE 12. In order to use the irrationality of $\theta(z)$, we define $b_{n}^{g}=1$ and $b_{n}^{h}=$ 0 , for all $n \geqslant 1$, and we choose $b_{n}^{s}$ later. Now we define $b_{n}, a_{n}$ as in Lemma 8 and denote by $(X, T),(\bar{X}, \bar{T})$ the pair of systems given there. Thus $T$ has one extra orbit in each length, compared to $T$, and the action of $\imath$ on $X$ has the effect of gluing together exactly one pair of orbits of each length.

Now we first take $b_{n}^{s}=\frac{1}{n} \sum_{d \mid n} \mu\left(\frac{n}{d}\right) 2^{d}-1$, for $n \geqslant 1$, so that $b_{n}$ is the number of orbits of length $n$ in any system with $2^{n}$ points of period $n$. Then

$$
\zeta_{\bar{T}}(z)=\frac{1}{1-2 z}
$$

while

$$
\mathrm{F}_{T}(n)=\sum_{d \mid n} d a_{d}=2^{n}+\sigma(n)
$$

so that

$$
\zeta_{T}(z)=\frac{1}{1-2 z} \theta(z)
$$

By the remarks above, this is not a rational function.

In the reverse direction, we take $b_{1}^{s}=1$ and $b_{n}^{s}=\frac{1}{n} \sum_{d \mid n} \mu\left(\frac{n}{d}\right) 2^{d}-2$, for $n \geqslant 2$, so that $a_{n}$ is the number of orbits of length $n$ in any system with $2^{n}+1$ points of period $n$. Then

while

$$
\zeta_{T}(z)=\frac{1}{(1-z)(1-2 z)}
$$

$$
\mathrm{F}_{\bar{T}}(n)=\sum_{d \mid n} d b_{d}=2^{n}+1-\sigma(n)
$$

so that

$$
\zeta_{\bar{T}}(z)=\frac{1}{(1-z)(1-2 z) \theta(z)},
$$

which is again irrational.

In fact, the zeta function in the previous example is worse than irrational. The function $1 / \theta(z)$ has integer coefficients and radius of convergence 1 , but is not rational. Thus, by the Pólya-Carlson Theorem (see $[\mathbf{2}, \mathbf{5}]$ ) it has the unit circle as natural boundary, and the function $\zeta_{\bar{T}}$ also has natural boundary here. Since the radius of convergence of $\zeta_{\bar{T}}$ is only $\frac{1}{2}$ this is perhaps not so interesting, but our final example shows that it is possible for the circle of convergence and the natural boundary of $\zeta_{\bar{T}}$ to coincide, even when $\zeta_{T}$ is rational.

EXAMPLE 13 . We begin by recursively defining an auxiliary sequence $\left(c_{n}\right)$ of non-negative integers by the following conditions:

- if $n=1$ or $n$ is prime, then $c_{n}=0$;

- if $n$ is composite then, for any prime $p$ dividing $n$,

$$
c_{n} \equiv c_{n / p} \quad\left(\bmod p^{\operatorname{ord}_{p}(n)}\right),
$$

and $n \leqslant c_{n}<2 n$.

Note that, by the Chinese Remainder Theorem, these conditions determine $\left(c_{n}\right)$ uniquely. Now set

$$
a_{n}=\frac{1}{n} \sum_{d \mid n} \mu\left(\frac{n}{d}\right) 2^{d}, \quad b_{n}=a_{n}+\frac{1}{n} \sum_{d \mid n} \mu\left(\frac{n}{d}\right) c_{d} 2^{d} .
$$


We assume for now that the sequences $\left(a_{n}\right),\left(b_{n}\right)$ are non-negative integers satisfying the conditions of Proposition 9 and denote by $(X, T),(\bar{X}, \bar{T})$ the systems given there with these numbers of orbits. Then

$$
\zeta_{T}(z)=\frac{1}{1-2 z}
$$

while

$$
z \frac{\zeta_{\bar{T}}^{\prime}(z)}{\zeta_{\bar{T}}(z)}=\frac{2 z}{1-2 z}+\sum_{n=1}^{\infty} c_{n} 2^{n} z^{n} .
$$

Now the integer sequence $\left(c_{n}\right)$ is not a linear recurrence sequence, since it is zero for all primes and non-zero for all composites, while the bound $c_{n}<2 n$ implies that the power series

$$
\sum_{n \geqslant 1} c_{n} z^{n}
$$

has radius of convergence 1. Hence it has a natural boundary on the unit circle, and we deduce that $z \frac{\zeta_{\bar{T}}^{\prime}(z)}{\zeta_{\bar{T}}(z)}$ has a natural boundary on the circle $|z|=\frac{1}{2}$. Thus $\zeta_{\bar{T}}$ also has a natural boundary here, since it has radius of convergence $\frac{1}{2}$.

It remains to show that $\left(a_{n}\right),\left(b_{n}\right)$ are non-negative integers and satisfy the conditions of Proposition 9. First, note that $a_{n}$ is the number of closed orbits of period $n$ of the tent map so that $\left(a_{n}\right)$ is a sequence of non-negative integers. Now we prove that the sequence $\left(b_{n}-a_{n}\right)$ is also a sequence of non-negative integers. First we must check that

$$
\sum_{d \mid n} \mu\left(\frac{n}{d}\right) c_{d} 2^{d}
$$

is a non-negative integer divisible by $n$, for all $n \geqslant 1$. To show that it is divisible by $n$, we show that it is divisible by $p^{\operatorname{ord}_{p}(n)}$, for each prime $p$ dividing $n$. For this, we use the following version of Euler's generalization of Fermat's Little Theorem: for any prime $p$ and integers $r, m$, with $p \mid m$, we have

$$
r^{m} \equiv r^{m / p} \quad\left(\bmod p^{\operatorname{ord}_{p}(m)}\right) .
$$

Thus, for any prime $p$ dividing $n$,

$$
\sum_{d \mid n} \mu\left(\frac{n}{d}\right) c_{d} 2^{d}=\sum_{\substack{d \mid n \\ p \nmid \frac{n}{d}}} \mu\left(\frac{n}{d}\right)\left(c_{d} 2^{d}-c_{d / p} 2^{d / p}\right) \equiv 0 \quad\left(\bmod p^{\operatorname{ord}_{p}(n)}\right),
$$

since, for any $d$ dividing $n$ with $p \nmid \frac{n}{d}$, we have $\operatorname{ord}_{p}(d)=\operatorname{ord}_{p}(n)$ and $c_{d} \equiv c_{d / p}$ $\left(\bmod p^{\operatorname{ord}_{p}(d)}\right)$ by construction.

For non-negativity, when $n$ is 1 or prime we have $b_{n}-a_{n}=0$. On the other hand, for $n$ composite, the bounds $c_{n} \geqslant n$ and $c_{d}<2 d \leqslant n$, for $d$ a divisor of $n$, imply that

$$
\sum_{d \mid n} \mu\left(\frac{n}{d}\right) c_{d} 2^{d}>n 2^{n}-\sum_{d \leqslant \frac{n}{2}} n 2^{d}>n\left(2^{n}-2^{\frac{n}{2}+1}\right)>0 .
$$

Finally, for $n$ composite, the same bounds show that

$$
b_{n}-a_{n}=\frac{1}{n} \sum_{d \mid n} \mu\left(\frac{n}{d}\right) c_{d} 2^{d}<\left(2^{n}+2^{\frac{n}{2}+1}\right)<2^{n+1},
$$


and, similarly,

$$
\frac{1}{n}\left(2^{n}-2^{\frac{n}{2}+1}\right)<a_{n}<\frac{1}{n}\left(2^{n}+2^{\frac{n}{2}+1}\right)<\frac{1}{n} 2^{n+1} .
$$

Thus, for $n \geqslant 6$, using that $2^{2 n}>(2 n+3) 2^{n+1}$, we have

$$
a_{2 n}>\frac{1}{2 n}\left(2^{2 n}-2^{n+1}\right)>\left(\frac{n+1}{n}\right) 2^{n+1}>\left(b_{n}-a_{n}\right)+a_{n}=b_{n} .
$$

Finally, one checks that $b_{4}=19<30=a_{8}$ and, since $b_{n}=a_{n}<a_{2 n}$ for $n$ prime, the conditions of Proposition 9 are satisfied with $N=2$.

\section{Concluding remarks and questions}

(1) The simple observation in [7] that for any sequence $\left(a_{n}\right)$ of non-negative integers there is a topological dynamical system $(X, T)$ with $\mathrm{O}_{T}(n)=a_{n}$ for all $n \geqslant 1$ was extended by Windsor [8], who showed that the map may be required to be an infinitely differentiable map on the 2-torus. Does Lemma 8 also have a smooth version, in which both maps and the involution are differentiable maps on a manifold?

(2) We have only considered quotients by an action of the group $C_{2}$. The same process makes sense if $(X, T)$ supports an action of some finite group $G$ commuting with $T$, and similar questions arise. In this setting the structure of the group plays a larger role, and other complications arise; this is explored in [9], where generalizations of Corollaries 7 and 10 are obtained. A particularly interesting sample problem is to understand a version of the relation (3) for other groups of symmetries.

(3) Achieving radius of convergence strictly smaller than 1 in Example 13 is important because with radius of convergence 1 the rational part of the Pólya-Carlson dichotomy is not particularly interesting: a rational Taylor series with integer coefficients and radius of convergence 1 has the form $\frac{p(z)}{\left(1-z^{a}\right)^{b}}$ for some polynomial $p$ with integer coefficients and integers $a, b \geqslant 0$. In our settings, this would correspond to dynamical systems in which the number of closed orbits of length $n$ is constant for large $n$.

\section{References}

[1] R. Bowen and O. E. Lanford, III., Zeta functions of restrictions of the shift transformation, in Global Analysis (Proc. Sympos. Pure Math., Vol. XIV, Berkeley, Calif., 1968), Amer. Math. Soc., Providence, R.I., 1970, 43-49.

[2] F. Carlson, Uber ganzwertige Funktionen, Math. Z. 11 (1921) 1-23.

[3] M. Einsiedler, E. Lindenstrauss, and T. Ward, Entropy in dynamics, to appear.

[4] G. Everest, A. van der Poorten, I. Shparlinski, and T. Ward, Recurrence sequences, Mathematical Surveys and Monographs, vol. 104, American Mathematical Society, Providence, RI, 2003.

[5] G. Pólya, Uber gewisse notwendige Determinantenkriterien für die Fortsetzbarkeit einer Potenzreihe, Math. Ann. 99 (1928) 687-706.

[6] G. Pólya and G. Szegö, Aufgaben und Lehrsätze aus der Analysis. Band II: Funktionentheorie. Nullstellen. Polynome. Determinanten. Zahlentheorie, Dritte berichtigte Auflage. Die Grundlehren der Mathematischen Wissenschaften, Band 20, Springer-Verlag, Berlin-New York, 1964.

[7] Y. Puri and T. Ward, Arithmetic and growth of periodic orbits, J. Integer Seq. 4 (2001) Article 01.2.1, 18 .

[8] A. J. Windsor, Smoothness is not an obstruction to realizability, Ergodic Theory Dynam. Systems 28 (2008) 1037-1041. 
[9] S. Zegowitz, Closed orbits in quotient systems, Ph.D. thesis, Univ. of East Anglia, 2014.

School of Mathematics, University of East Anglia, Norwich Research Park, NorWICH UK, NR4 7TJ

E-mail address: shaun.stevens@uea.ac.uk

Executive Office, Durham University, Durham UK, DH1 3LE

E-mail address: t.b.ward@durham.ac.uk

College of Engineering, Mathematics and Physical Sciences, University of Exeter, North Park RoAd, Exeter UK, EX4 4QF

E-mail address: S.H.Zegowitz@exeter.ac.uk 